\title{
Hemorroidectomía cerrada y semicerrada: Estudio prospectivo aleatorizado*
}

\author{
Drs. RODRIGO AZOLAS M. ${ }^{1,2}$ M., RICARDO VILLALÓN C. ${ }^{1}$, STEFAN DANILLA E. ${ }^{3}$, \\ ANDREA HASBÚN N. ${ }^{1}$, FRANCISCO GATICA F. ${ }^{1}$, JORGE SALAMANCA B. ${ }^{1}$
}

1 Servicio y Departamento de Cirugía Hospital Barros Luco Trudeau. Campus Sur Facultad de Medicina Universidad de Chile.

2 Departamento de Cirugía Hospital Clínico Universidad de Chile.

3 Epidemiología Clínica Universidad de los Andes. Santiago, Chile.

\begin{abstract}
\section{Prospective randomized comparison of open and closed hemorrhoidectomy}

Background: Grade III and IV internal hemorrhoids have a surgical indication. However there is no consensus about the best surgical procedure since all techniques have drawbacks, especially in terms of pain. Aim: To compare the results of open and closed hemorrhoidectomy. Material and Methods: Patients with grade III or IV internal hemorrhoids were randomly assigned to be treated with open or closed hemorrhoidectomy. Postoperative pain and complications were evaluated during 21 days in the postoperative period. Results: Twenty four patients were assigned to each treatment group. No differences between groups were observed for postoperative pain, complications, operative time or time required to go back to work. Ninety five percent of patients considered the ambulatory surgical procedure as good or very good. Conclusions: No differences between open and close hemorrhoidectomy were observed in this series of patients.
\end{abstract}

Key words: Hemorrhoidectomy, internal hemorrhoids, pain.

\section{Resumen}

La enfermedad hemorroidaria interna grados III y IV se trata quirúrgicamente; actualmente no existe un gold standar, ya que las diferentes técnicas tienen sus inconvenientes, sobre todo en relación al dolor. El objetivo del presente estudio es comparar los resultados de la hemorroidectomía cerrada $\mathrm{v} / \mathrm{s}$ la semicerrada en términos de dolor, complicaciones, tiempo operatorio y cicatrización. Pacientes y Método: Ensayo clínico controlado aleatorizado; se incluyeron 48 pacientes con hemorroides internos G III y IV operados en forma ambulatoria, se evaluó el dolor a través de la escala visual análoga (EVA) además del sangrado, infección, cicatrización, tiempo operatorio, reintegro laboral y satisfacción con respecto al procedimiento. Resultados: En este estudio se incluyeron 48 pacientes para hemorroidectomías, 24 cerradas y 24 semicerradas, ambos grupos fueron comparables. En relación al dolor no hubo diferencias entre ambos grupos tanto como para la medición de la escala EVA $(p=0,1768)$, como para la necesidad de rescate analgésico $(p=0,3290)$. No hubo diferencias en relación a las complicaciones, cicatrización, tiempo operatorio y reintegro laboral. El $95 \%$ de los pacientes consideró que la cirugía ambulatoria era buena y muy buena. Conclusiones: No existen diferencias significativas entre ambas técnicas y la cirugía ambulatoria es un procedimiento seguro y bien aceptado.

Palabras clave: Hemorroidectomía cerrada, hemorroidectomía semicerrada, complicaciones.

\footnotetext{
Correspondencia: Dr. Rodrigo Azolas M.

Basel \#4666, Casa N, Lo Barnechea, Santiago, Chile.

E-mail: rodrigoazolas@gmail.com
}

*Recibido el 9 de Octubre de 2009 y aceptado para publicación el 3 de Noviembre de 2009. 


\section{Introducción}

La hemorroidectomía se considera un procedimiento eficaz pero doloroso para el tratamiento de la enfermedad hemorroidal, cuando el tratamiento médico ha fracasado.

Actualmente el tratamiento quirúrgico se reserva para las hemorroides internos grados III y IV. A través del tiempo se han descrito muchas técnicas quirúrgicas diferentes, sin embargo, ninguna de ellas ha sido aceptada como gold standar ${ }^{1}$. Los principios quirúrgicos comprenden la eliminación de los plexos que prolapsan, a veces combinada con la reubicación del epitelio escamoso ${ }^{2,3}$. Se han realizado varios estudios comparando la hemorroidectomía cerrada con la abierta y la submucosa con la abierta, encontrándose diferencias en la cicatrización, y en relación al prurito y la descarga. Para algunos autores norteamericanos la hemorroidectomía cerrada podría ser menos dolorosa ${ }^{4}$.

La técnica cerrada consiste en resecar el paquete hemorroidal y reparar el defecto en su totalidad, en cambio en la semicerrada se repara el defecto hasta el margen anal.

El objetivo del presente estudio prospectivo aleatorizado, es comparar los resultados de la hemorroidectomía cerrada con la semicerrada, en términos de dolor, complicaciones, tiempo operatorio, cicatrización, reintegro laboral y evaluación de la cirugía ambulatoria.

\section{Material y Método}

Ensayo clínico controlado aleatorizado, realizado en la Unidad de Cirugía Ambulatoria del Hospital Barros Luco Trudeau (Centro terciario) entre marzo de 2006 y marzo de 2007. A todos los pacientes se les solicitó consentimiento informado antes de ser incorporados a este estudio.

\section{Criterios de Inclusión}

Pacientes con diagnóstico de Hemorroides Internos G III a IV que se operaron en forma ambulatoria.

\section{Criterios de Exclusión}

Pacientes con diagnóstico asociado de fisura anal, fístula anorrectal, absceso anorrectal, enfermedad inflamatoria, condilomas o cáncer de ano y que no cumplan con los criterios para cirugía ambulatoria.

Se incluyeron 48 pacientes que se separan en forma computacional en dos grupos.

Un grupo fue tratado con hemorroidectomía cerrada (HC) y el otro semicerrada (HS). Todos los pacientes recibieron anestesia raquídea y se realizó infiltración de cada paquete hemorroidal con adrenalina 1:200 asociado y $2 \mathrm{mg}$ de bupivacaina. Disección por separado con electrobisturí, ligadura del paquete en su base y reparación del defecto mucoso con puntos discontinuos de Vicryl ${ }^{\circledR} 3-0$. El cierre fue completo en la $\mathrm{HC}$ y hasta el margen anal en la HS.

Los pacientes desconocían la técnica efectuada.

Se controló el tiempo operatorio en minutos.

Todos los pacientes fueron dados de alta a las 6 u $8 \mathrm{hrs}$ post operatorias con las siguientes indicaciones:

1er día postop. Calor húmedo local y reposo relativo.

Régimen con abundante fibra más líquidos.

Vaselina líquida 1 cucharada en la noche.

Aseo perianal post defecación.

Baño de asiento caliente (BAC) a partir del 20 día, c/6 u 8 hrs.

Diclofenaco $50 \mathrm{mg}$ c/8h vía oral.

Acetaminofeno $325 \mathrm{mg}+$ Cloridrato de tramadol $37,5 \mathrm{mg}$ (Zaldiar $\left.{ }^{\circledR}\right) 1$ comp en caso de dolor.

Control telefónico a las $24 \mathrm{hrs}$ del postoperatorio por enfermera de la unidad.

Evaluación del dolor: Se midió utilizando la escala visual análoga (EVA), previa utilización de analgesia, 3 veces al día durante los primeros 5 días, y una vez al día durante los siguientes 21 días. Se registró también la cantidad de analgésicos de rescate que fue utilizado diariamente.

Evaluación de complicaciones: Se registraron durante los controles médicos realizados a los 5, 14 y 21 días, consignando la presencia de: retención urinaria, sangrado, infección y grado de cicatrización.

A las 6 semanas, vía telefónica, se preguntó sobre el tiempo de reposo antes de iniciar sus actividades normales y el grado de satisfacción de la cirugía ambulatoria, siendo 1 muy bueno, 2 bueno, 3 regular y 4 malo.

\section{Análisis estadístico:}

Tamaño muestral: Para la escala visual análoga se determinan 24 pacientes por grupo teniendo un poder de un $80 \%$. Para detectar una variación, entre los grupos de 0,391 , cuando el nivel de significancia es de 0,050 .

Para la analgesia de rescate se determinan 24 pacientes por grupo teniendo un poder de un $86 \%$. Para detectar una variación, entre los grupos de 0,033 , cuando el nivel de significancia es de 0,050 . (nQuery 4.0 R).

Análisis: Para detectar diferencias estadísticas entre los grupos en mediciones repetidas, se utilizaron ecuaciones de estimación generalizadas. Se usó 
distribución Gaussiana para los datos continuos y ordinales, y distribución binaria para datos ordinales.

Para comparación simple entre los grupos se usó t-test y test de proporciones. Para significación estadística usamos test de 2 colas, alfa de 0,0500 (Stata 9.0, Stata Corporation, TX, USA).

Reporte de datos: Las variables continuas se expresan como promedio y desviación estandar (DS), las ordinales como promedio y rango, y las binarias como porcentaje.

\section{Resultados}

En este estudio se incluyeron 48 pacientes para hemorroidectomías, 24 cerradas y 24 semicerradas, ambos grupos fueron comparables, $62,5 \%$ de pacientes de sexo femenino para cada grupo $(\mathrm{p}=1,0)$ y un promedio de edad de 49,3 HC y 43,5 HS ( $p=0,19$ ) (Tabla 1).

En relación al dolor no hubo diferencias entre ambos grupos, tanto para la medición de la escala EVA (p $=0,1768)$, como para la necesidad de rescate analgésico $(\mathrm{p}=0,3290)$ (Figuras 1 y 2).

En relación a la evaluación de las complicaciones, ningún paciente presentó retención urinaria, el $75 \%$ de los pacientes en los cuales se realizó HS sangraron la primera semana, uno de estos requirió hospitalización en el postoperatorio inmediato, en relación al $62,5 \%$ de las HC, el sangrado fue disminuyendo hasta un $12,5 \%$ en la HS y un $8,3 \%$ en las $\mathrm{HC}$ a la tercera semana, la diferencias para ambos grupos no son estadísticamente significativas $(\mathrm{p}$ $=0,6130)$. En relación a la infección se presentaron 3 casos, 2 de los cuales correspondieron a la HS y 1 a la HC, (p $=0,6301)$ (Tabla 2).

El proceso de cicatrización se comenzó a observar a partir de la tercera semana, momento en el cual un $41,7 \%$ de las $\mathrm{HC}$ y un $33,3 \%$ de las HS presentaron cicatrización completa $(\mathrm{p}=$ 0,5505).

En relación al tiempo operatorio y a la reincorporación a la actividad laboral no se encontró diferencia significativa
Tabla 1. Características básicas de la muestra

\begin{tabular}{lccc}
\hline Características & $\begin{array}{c}\text { Semicerrada } \\
\mathbf{n = 2 4}\end{array}$ & $\begin{array}{c}\text { Cerrada } \\
\mathbf{n = 2 4}\end{array}$ & p-value \\
\hline Edad & $49,3 \pm 10,1$ & $45,0 \pm 12,4$ & 0,1921 \\
Sexo (Femenino) & $62,5 \%$ & $62,5 \%$ & 1,0000 \\
\hline
\end{tabular}

Tabla 2. Complicaciones postoperatorias

\begin{tabular}{lccc}
\hline \multirow{2}{*}{ Complicaciones } & Semana post & Semicerrada & Cerrada \\
\hline Sangrado & 1 & 75,0 & 62,5 \\
& 2 & 41,7 & 37,5 \\
\multirow{2}{*}{ Infección } & 3 & 8,3 & 12,5 \\
& 1 & 4,2 & 4,2 \\
Cicatrización & 2 & 4,2 & 0,0 \\
& 3 & 0,0 & 0,0 \\
& 1 & 0,0 & 0,0 \\
& 2 & 0,0 & 0,0 \\
& 3 & 33,3 & 41,7 \\
\hline
\end{tabular}

Tabla 3. Nivel de satisfacción a cirugía ambulatoria

\begin{tabular}{lccrr}
\hline Nivel & Semicerrada (\%) & Cerrada (\%) & \multicolumn{2}{c}{ Total (\%) } \\
\hline 1 Muy Bueno & $11(45,8)$ & $10(41,6)$ & $21(43,75)$ \\
2 Bueno & $12 \quad(50)$ & $13(54,1)$ & 25 & $(52)$ \\
3 Regular & $1 \quad(4,2)$ & $1(4,2)$ & 2 & $(4,25)$ \\
4 Malo & 0 & 0 & 0 \\
\hline
\end{tabular}

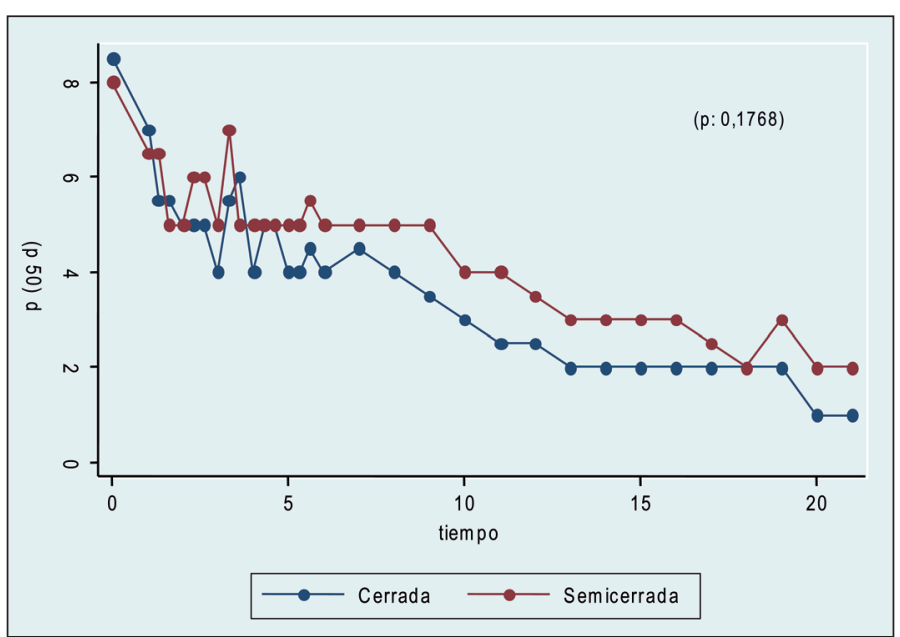

Figura 1. Evolución de la escala visual análoga. 


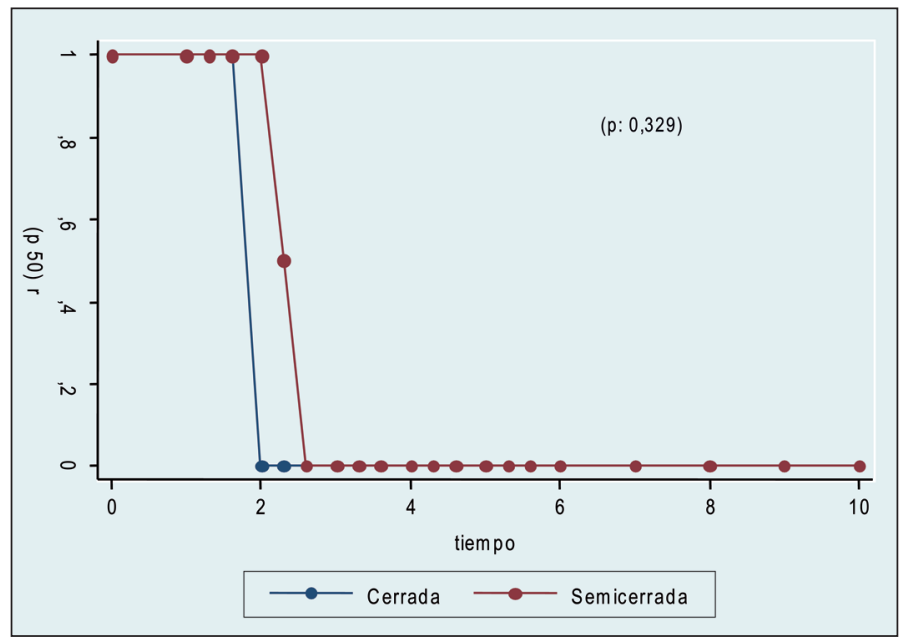

Figura 2. Rescate analgésico en el tiempo.

para ambas técnicas.

El nivel de satisfacción de los pacientes en relación a la cirugía ambulatoria fue de un 95\% para la calificación entre muy bueno y bueno (Tabla 3 ).

\section{Discusión}

La patología hemorroidal es uno de los principales motivos de consulta en coloproctología y su resolución quirúrgica puede hacerse realizando una cirugía abierta o cerrada. Tradicionalmente se ha dicho que la cirugía abierta tiene el inconveniente del escape, el prurito y el mayor tiempo de cicatrización al no repararse el defecto mucoso, y la cirugía cerrada sería un poco más dolorosa por los puntos de la piel, y a la semana un gran porcentaje se abre, es por esto que el realizar una hemorrroidectomía semicerrada, reparando el defecto mucoso hasta el margen anal dejando la piel abierta, podría mejorar las molestias de la cirugía abierta y disminuir el dolor post operatorio.

Con respecto al dolor se observó que no existían diferencias significativas, al igual que en los trabajos realizados por Carapeti ${ }^{5}$ y Arbman ${ }^{6}$; esto se evaluó a través de la escala de EVA y de la cantidad de analgésicos requeridos durante el postoperatorio, a pesar de no existir diferencias significativas se puede observar que en la HS el dolor es algo menor.

Ningún paciente presentó retención urinaria, en concordancia con lo reportado por Hoff $\mathrm{SD}^{7}$, donde se le atribuye un rol importante a la cirugía ambulatoria, en la disminución de esta complicación, ya que el confort del hogar favorecería la micción espontánea del paciente.
En concordancia con lo publicado comparando técnicas abiertas con cerradas, nosotros no encontramos diferencia significativa en relación al sangrado post operatorio. Se infectaron 2 pacientes en la HS y 1 paciente en la HC (NS), que respondieron favorablemente al uso de antibióticos orales, esta complicación no se evalúa en ningún trabajo de los revisados.

A diferencia de Gencosmanoglu ${ }^{8}$, no obtuvimos mayor tiempo operatorio entre las técnicas utilizadas.

La reincorporación laboral tampoco presentó diferencia entre una y otra técnica y ocurrió en promedio al día 30 de postoperado.

Destaca al alto grado de satisfacción referido para la realización del procedimiento en forma ambulatoria, $95 \%$ lo calificó entre muy bueno y bueno.

\section{Conclusiones}

Los resultados de este estudio prospectivo randomizado, demuestran que no existen diferencias significativas para la hemorroidectomía cerrada y semicerrada, en relación al dolor, complicaciones postoperatorias, cicatrización y tiempo operatorio. El procedimiento puede ser realizado en forma ambulatoria, de manera segura y con un alto grado de satisfacción por parte de los pacientes.

\section{Referencias}

1. Parks AG. The surgical of haemorrhoids. Br J Surg 1956; 43: 337-357. 
2. Milligan ET, Morgan CN, Jones LE, Officer R. Surgical anatomy of the anal canal, and the operative treatment of hemorrhoids. Lancet 1937; 2: 1119-1124.

3. Ferguson JA, Heaton JR. Closed hemorrhoidectomy. Dis. Colon Rectum 1959; 2: 176-179.

4. Hosch SB, Knoefel WT, Pichlmeier U, Schulze V, Busch C, Gawad K, et al. Surgical treatment of piles. Prospective, randomized study of Parks vs MilliganMorgan hemorrhoidectomy. Dis Colon Rectum 1998; 41: 159-164.

5. Carapeti EA, Kamm MA, McDonald PJ, Chadwick SJD, Phillips KS. Randomized trial of open versus closed day-case haemorrhoidectomy. Br J Surg 1999; 86: 612-613.
6. Arbman G, Krook H, Haapaniemi S. Closed v/s Open Hemorrhoidectomy - Is there any difference. Dis Colon Rectum 2000; 43: 31-34.

7. Hoff SD, Bailey HR, Butts DR, Max E, Smith K, Zamora L, Skakun G. Ambulatory surgical hemorrhoidectomy- a solution to postoperative urinary retention? Dis Colon Rectum 1994; 37: 1142-1144.

8. Gencosmanoglu R, Sad O, Koc D, Inceoglu R. Hemorrhoidectomy: Open or Closed Technique. Dis Colon Rectum 2002; 45: 70-75.

9. Mortensen PE, Olsen J, Pedersen IK, Christiansen J. A randomized study on haemorrhoidectomy combined with anal dilatation. Dis Colon Rectum 1987; 30: 755 757. 